\title{
Single Track Effects, Biostack and Risk Assessment
}

\author{
Stanley B. Curtis \\ Life Sciences Division \\ Lawrence Berkeley Laboratory \\ University of California \\ Berkeley, California 94720
}

October 1992

Ceremonial Address presented at "20 Years of BIOSTACK", the Colloquium honoring Professor Dr. Horst Bücker, at DLR, Koln-Porz, Germany, on Octuber 9, 1992.

This work was supported by the National Aeronautics and Space Administration under Order No. T-9310R, and by The NASA Specialized Center of Research and Training (NSCORT) through the U.S. Department of Energy under Contract No. DE-ACO3-76SFO0098.

\section{MASTER}




\title{
Single Track Effects, Biostack and Risk Assessment
}

\author{
Stanley B. Curtis \\ Life Sciences Division \\ Lawrence Berkeley Laboratory \\ University of California \\ Berkeley, CA 94720 U.S.A.
}

\section{Abstract}

The scientific career of Ptof. Bucker has spanned a very exciting period in the fledgling science of Space Radiation Biology. The capability for placing biological objects in space was developed, and the methods for properly packaging, retrieving, and analyzing them were worked out. Meaningful results on the effects of radiation were obtained for the first time. In fact, many of the successful techniques and methodologies for handling biological samples were developed in Prof. Bucker's laboratories, as attested by the extensive Biostack program. He was the first to suggest and successfully carry out experiments in space directly aimed at measuring effects of single tracks of high-energy heavy galactic cosmic rays by specifically identifying whether or not the object had been hit by a heavy particle track. Because the "hit" frequencies of heavy galactic cosmic rays to cell nuclei in the bodies of space travelers will be low, it is expected that any effects to humans on the cellular level will be dominated by single-track thaversals. This includes the most important generally recognized late effect of space radiation exposure: radiation-induced cancer.

This talk addresses the single-track nature of the space radiation environment, and points out the importance of single "hits" in the evaluation of radiation risk for long-term missions occurring outside the earth's magnetic field. A short review is made of biological objects found to show increased effects when "hit" by a single heavy charged-particle in space. A brief discussion is given of the most provocative results from the bacterial spore B. subtilis. experimental evidence that tracks can affect biological systems at much larger distances from the trajectory than previously suspected, and that the resultant inactivation cross section in space calculated for this system is very large. When taken at face value, the implication of these results, when compared to those from experiments performed at ground-based accelerators with beams at low energies in the same LET range, is that high-energy particles can exert their influence a surprising distance from their trajectory and the inactivation cross sections are some twenty times larger than expecied. Clearly, beams from high-energy heavy-ion accelerators should be used to confirm these results.

For those end points that can also be caused by low-LET beams such as highenergy protons, it is important to measure their action cross sections as well. The ratio of the cross sections for a high-LET beam to that of a low-LET beam is an interesting experimental ratio and, we suggest, of more intrinsic interest than the RBE. It is a measure of the "biological" importance of one particle type relative to another particle type. This ratio will be introduced and given the name RPPE (Relative Per Particle Effectiveness). Values of RPPE have appeared in the literature and will be discussed. A rather well-known value of this quantity $(13,520)$ has been suggested for the RPPE of high-energy iron ions to high-energy protons. This value was suggested by Letaw et al. [Nature 330, 709-710 (1987)]; we will call it the Letaw limit. It will be discussed in terms of the importance of the heavy-ion component vs. the light-ion component of the galactic cosmic rays. It is also pointed out, however, that there may be unique effects from single tracks of heavy ions that do not occur from light-ion tracks. For such effects, the concepts of both RBE and RPPE lose their meaning. 


\section{Introduction}

It is a distinct honor to address this international gathering to celebrate the distinguished career of Professor Horst Bücker. May I begin by offering my congratulations to you, Professor Bücker, for this outstanding accomplishment. I hope that those of us who will carry on in the field will continue with as much energy and enthusiasm as you have shown. I also bring greetings from your friends and colleagues in the United States who were unable to attend. In particular, Professor Benton told me to convey his congratulations to you and to relay his regret that he was especially sorry not to be able to be here today.

The effects of single tracks of heavy galactic cosmic rays on biological systems is a fascinating and challenging field of study. It is hard to imagine a more truly interdisciplinary enterprise, with such fields as physics, chemistry, biochemistry, molecular biology, genetics and tissue biology all playing a prominent role. Other readily identifiable subfields such as, biophysics, radiation chemistry, radiation biology, neurobiology, carcinogenesis, and perhaps even gravitational biology in one way or another attempt to bridge the gaps between the main disciplines to lead ultimately to the goal of radiation risk assessment. This interdisciplinary character has always intrigued me, and, in fact, was a strong inducement for me to enter the field some thirty years ago. I find it a continual pleasure to have the upportunity to learn something new in neighboring and related fields. I know, Professor Bücker, that you share this excitement in the interdisciplinary character of the field.

\section{First to Correlate Single Tracks in Space with Biological Bffects}

It was actually a quarter of a century ago, that Professor Bücker initiated the Biostack program at the University of Frankfurt. The objectives of the first Biostack experiments which were eventually flown aboard Apollo 16 and 17 were expressly: "to study the biological effects of individual heavy cosmic particles of high-energy loss (HZE) not available on earth; to study the influence of additional space flight factors; to get some knowledge on the mechanism by which HZE particles damage biological materials; to get some information on the spectrum of charge and energy of the cosmic ions in the spacecraft; to estimate the radiation hazards for man in space" (Buicker, 1974). The capability for placing biological objects in space was developed in his laboratories, and the means were worked out for properly packaging, retrieving, and analyzing them by determining with appropriately placed single-track detectors which biological organisms had been hit by a highly ionizing heavy charged particle. He was, in fact, the first to suggest and successfully carry out experiments in space directly aimed at measuring effects of single tracks of high-energy heavy galactic cosmic rays by specifically identifying whether or not the biological object had been hit by a heavy particle track. The Biostack concept and methodology have provided a rich source of single-track data, and many of the results will be discussed later tolay in this colloquium. He is to be congratulated that, through a truly international and, I might add, interdisciplinary collaboration, a large amount of data on biological effects of heavy charged-particles has been collected both in space and at heavy-ion accelerators. 


\section{Review of All Single Track Results in Space}

A survey of the results recently published by Dr. Gerda Horneck (Horneck, 1992) has shown that the evidence appears irrefutable that single traversals of highenergy heavy galactic cosmic rays do enhance measurable effects of many different types of resting biological systems, namely, Bacillus subtilis spores, Arabidopsis thaliana seeds, Nicotiana tabaccum seeds, Lactuca sativa seeds, Artemia salina cysts, Tribolium confusium eggs, and Carausius morosus eggs. In order to try to get a quantitative idea of the magnitude of single-particle effects on the biological systems exposed in space, I have calculated the ratio of effects from "hit" to "non-hit" biological objects flown using the data as collected in the recent published review by Dr. Horneck (Horneck, 1992). These ratios are shown in Fig. 1. Here the effects were assumed to be due to random events. From the fractions of hit and non-hit samples with no effect, mean values for the number of "biologically meaningful events per sample" can be calculated assuming such events are Poissonly distributed throughout the hit and non-hit populations, respectively. The ratio plotted is the ratio of the mean numbers of "biologically meaningful events per sample" for the hit to the non-hit samples. A value one for the ratio would indicate that a hit sample was found experimentally to have no higher probability of showing the effect than a non-hit sample. We see that the majority of biological systems show values greater than one. Forty-three percent of the systems gave ratios greater than two while $17 \%$ gave ratios less than one. The four systems and end points identified in the graph gave ratios of 6 or greater. Not shown in the graph are several end points for which there were no measureable effects for non-hit samples. For those, of course, no ratios can be calculated. These results taken together provide convincing evidence that single high-energy galactic-cosmic-ray track effects can be measured in resting biological systems.

\section{A Provocative Result: Long-range Track Effects on Bacterial Spores}

I now want to mention briefly the most provocative set of experimental results that, in my opinion, have come from the program. Experiments using the bacterial spore Bacillus subtilis have produced results that suggest that high-energy galactic cosmic rays (in the energy range 20-300 Mev/amu, mostly iron ions) can affect biological objects at considerable distances from the track trajectory (up to $3.8 \mu \mathrm{m}$ ), much farther than distances expected to be effected by the delta-ray cloud surrounding the tracks. The resultant inactivation cross section calculated by single-particle-effect analysis was $5.24 \mu \mathrm{m}^{2}$, some twenty times the value of the cross sectional area of the spore itself and considerably higher than inactivation cross sections measured at accelerators either from fluence effect curves or from the single-particle effect analysis (e.g., Horneck et al, 1989). The fascinating implication of these results is that a highenergy galactic cosmic ray can exert its effect considerably further away than might be expected from the delta rays alone. Additional mechanisms such as shock waves or thermophysical events have been suggested as being the cause of these long range effects. I have no new suggestions as to mechanisms or alternative interpretations of the data. I will only say that such unexpected results are what keeps this field of science interesting and exciting. I suggest continue to keep an open mind, always keep looking for more mundane explanations, and we will all be interested in the 
results of experiments with this system on high-energy iron beams at a high-energy heavy-ion accelerator.

\section{Link of Single Tracks to Risk Assessment}

We now ask the question: what does all this have to do with assessing the radiation risk from galactic cosmic rays? The answer lies in the relation these singletrack effects have to causing late effects such as cancer within the human body. Calculations have shown that galactic cosmic ray intensities are such that, on the cellular level, single-cell-traversals will dominate (Curtis and Letaw, 1989). For example, under a realistic shielding configuration over a three-year mission outside the geomagnetosphere at solar minimum, the mean hit frequency for cell nuclei 100 $\mu \mathrm{m}^{2}$ in area is 0.7 for carbon ions, 0.5 for oxygen ions, and 0.03 for iron ions. This means that although a considerable fraction of cells will be hit, only a very small fraction will receive more than one or a few hits. The majority of cell nuclei that are hit will only receive one. This emphasizes the importance of single-rack effects such as those measured in the Biostack program. Since it is generally believed that important molecular events (e.g., a mutation or deletion) occur within a single cell to initiate it on the pathway to malignancy, it is clear that effects on single cells are important.

The most pressing question regarding risk assessment becomes: what is the relative risk of causing a carcinogenic or other damaging event by traversals of the different types of particles found in the galactic cosmic radiation? Because of the single-track nature of the radiation environment, this question has more relevance than a consideration of the Relative Biological Effectiveness (RBE). This question has been addressed in the literature (Curtis et al, 1992) and a risk methodology involving "risk cross sections" instead of Quality Factors has been introduced. The idea here is to relate the biological effect directly to the fluence of particles instead of to the absorbed dose. Thus, the risk cross section, $\boldsymbol{F}_{\mathrm{i}}(\mathrm{L})$, is defined in analogy to the inactivation cross sections discussed above:

$$
P=\sum_{i} \int F_{i}(L) \phi_{i}(L) d L
$$

Here $P$ is the total probability (risk) of the end point of interest and $F_{i}(L)$ is the probability per unit fluence (the fluence-related risk coefficient or risk cross section) of the end point at LET, $L$, and $\phi_{i}(L)$ is the differential fluence spectrum in number of particles of type $i$ per unit area between $L$ and $L+d L$ at the point of interest. The summation is over all the different particle types in the spectrum.

Conventionally, the risk, $\mathbf{R}_{\mathbf{i}}$, from the ith particle type is defined as follows:

$$
R_{i}=R_{\gamma} H_{i}=R_{\gamma} \int Q(L) L \phi_{i}(L) d L
$$

where $R_{\boldsymbol{\gamma}}$ is the risk per unit dose equivalent from gamma rays and $\mathbf{H}_{\mathbf{i}}$ is the dose equivalent from the ith particle type. $Q(L)$ is the Quality Factor as a function of the 
LET, $L$, and $\phi_{i}(L)$ is the differential fluence spectrum of the ith particle type between $\mathbf{L}$ and $\mathbf{L}+\mathbf{d} \mathbf{L}$. From our definition of risk cross section above, we may write the risk of the ith particle type:

$$
R_{i}=\int F_{i}(L) \phi_{i}(L) d L
$$

Comparing equations (2) and (3), we see that we can write a conventional risk cross section in terms of the conventional Quality Factor:

$$
\mathbf{F}_{c}(\mathbf{L})=\mathbf{R}_{\boldsymbol{\gamma}} \mathbf{Q}(\mathbf{L}) \mathbf{L}
$$

We note that this is a universal function of LET; that is, its value does not depend on the particle type, but only on its LET. In other words, all particle types of the same LET will have the same conventional risk cross section. Because of differences in track structure of the different types of particles at the same LET, this assumption might be invalid at least in some ranges of LET. We do not yet have the appropriate data to evaluate the effect of this approximation.

\section{Definition of Relative Per Particle Effectiveness (RPPE)}

This line of reasoning leads to the idea of defining a quantity that is analogous to the Quality Factor (the ratio of doses to produce the same biological effect) but is instead the ratio of fluences $\left(\phi_{j} / \phi_{i}\right)$ to produce the same biological effect or, at low fluences where the single-particle assumption is valid, simply the (reciprocal) ratio of the risk cross sections, $\mathbf{F}_{\mathrm{i}}\left(\mathrm{L}_{\mathrm{i}}\right) / \mathbf{F}_{\mathrm{j}}\left(\mathrm{L}_{\mathrm{j}}\right)$, where $\mathrm{i}$ and $\mathrm{j}$ are two particle types. Because of its conceptual importance, we will give this ratio the name Relative Per Particle Effectiveness (RPPE).

Using equation 4 , the ratio of conventional cross sections, which is the "conventional" RPPE, is:

$$
\operatorname{RPPE}_{c, \mathrm{i}, \mathrm{j}}=\mathbf{Q}\left(\mathrm{L}_{\mathrm{i}}\right) \mathrm{L}_{\mathbf{i}} / \mathrm{Q}\left(\mathrm{L}_{\mathbf{j}}\right) \mathrm{L}_{\mathbf{j}}
$$

where $\mathrm{i}$ and $\mathrm{j}$ denote the two particle types in question. The appropriate interpretation of the RPPE is that it is the relative risk per particle for the risk in question. It is correct to state that, in conventional terms, a particle of type $i$ has a risk associated with it that is $\mathrm{RPPE}_{c, i, j}$ times the risk associated with particle of type $j$.

The use of this ratio is not new and has been commented upon in the past by several authors. In particular, it was discussed in a paper by Letaw et al (1987), in which the relative risk posed by the high-energy iron component was compared to that posed by the proton component of the galactic cosmic rays. I am presently taking this opportunity now to suggest that, because of its conceptual importance, this ratio be given a formal name. 


\section{The Letaw Limit}

In the paper by Letaw et al, the assumption was made that both the iron ions ard protons were minimum ionizing, i.e., that their LET's differed by the ratio of the square of their charges: 676 to 1 . The protons were assumed to have a Quality Factor of one, commensurate with their LET of about $0.2 \mathrm{keV} / \mu \mathrm{m}$. The iron ions were assumed to have a Quality Factor of 20, commensurate with their LET of about 140 $\mathrm{keV} / \mu \mathrm{m}$. From equation 5, we see that the $\mathrm{RPPE}_{\mathrm{c}, \mathrm{Fe}, \mathrm{p}}$ is $20 \times 676=13,520$. It is correct to interpret this in conventional terms as the relative per particle efficiency for causing cancer, that is, a high-energy iron ion is 13,520 times as probable to cause cancer in crossing a cell or a given number of cells as a proton. Whether this is truly the case is one of the major unanswered questions in galactic cosmic ray risk assessment. A modification has been suggested to account for the increase of effect from the nuclear interactions of high energy protons (Wilson et al, 1990). It was found that "target fragmentation", occurring in a nuclear collision between protons and a nucleus in tissue, produces low-energy high-LET tracks that increase the conventional proton risk cross section by about a factor of two in the energy region of 1 $\mathrm{GeV} /$ nucleon and above. This effect was found to be less important at lower energies because (1) fewer channels are open for the emission of nuclear secondary particles, i.e., the total nuclear cross section is less, and (2) the LET of the proton increases at lower energies and tends to overshadow nuclear effects. It is interesting to note that for the heavier galactic cosmic ray components, nuclear cross sections for target fragments are negligible compared to the effect due to ionization. Therefore, we may consider the calculated conventional RPPE of 13,520 as an upper limit to the relative effectiveness per particle for this particle-pair. Assuming a factor of two increase in the proton risk cross section from these nuclear target fragmentation effects (a conservatively large estimate), the conventional RPPE for iron to protons decreases to 6,800 for the iron-proton particle pair. Although the conventional upper limits, which we will call the Letaw limits, are easy to calculate for any particle-pair, given a Quality Factor dependence on LET, it is very difficult to obtain a feeling for more realistic values. I would suggest that one of the crucial tasks in the next decade is to arrive at an overall understanding of risk cross sections for the various components of the galactic cosmic rays. A concerted effort must be made to determine the cancer risk cross sections for all the major components of the galactic cosmic rays along with their dependence on LET (or energy). Such questions as whether cross sections of different particle types at the same LET have significantly different cross sections due to differences in track structure should also be carefully studied. Major uncertainties remain in picking the appropriate biological systems that mimic the response of cellsat-risk in the human body. Much must be done at the molecular, cellular, tissue, organ and whole animal levels to assure that all aspects of the human response are appropriately evaluated. This brings me back to my earlier remarks on the interdisciplinary nature of this enterprise. It is still an exciting area of research. There is much to do and much to learn. The effort must involve strong cooperation between scientists in many disciplines, not only within the biological community, but also across the physics and chemistry communities as well. I cannot refrain at this point from remarking that interlaced throughout the studies, the mechanistic modeler will play a major role in integrating the experimental results into models of human response to radiation, because it will remain impossible to accumulate enough 
statistically meaningful data directly from space travelers exposed to the galactic cosmic rays for many decades to come.

\section{Inadequacy of RBE and RPPE}

Finally, it should be remembered that it has not been established experimentally that all risks from the galactic cosmic rays have been identified. There are intriguing hints that for certain sensitive biological processes there is a synergistic effect between radiation and microgravity. Some of these results have come from the Biostack Program. It has been suggested over the years that there is a distinct possibility that certain yet-to-be-discovered effects from the highly ionizing component of the galactic cosmic rays might occur that have no counterpart in exposure to more lightly ionizing radiation. Perhaps we have seen evidence of this in the Biostack Program as well. If such effects occur, for instance as an accumulation of damage over a long time in the central nervous system, the concepts of both RBE and RPPE lose their meaning, since the denominators in each case vanish. The risk cross section concept, however, retains its usefulness because it is simply the risk per unit fluence and no relationship with a low-LET standard is necessary, while the conventional idea of Quality Factor will clearly have to be abandoned, since it is related to the RBE, and the ability to measure an effect at low LET is a necessary condition for its validity.

\section{Concluding Remarks}

So, Professor Bücker, it has been a pleasure to share these thoughts with you and your colleagues assembled here in your honor today. I know everyone joins me in wishing you the best in the years ahead. I personally hope you will continue to contribute your expertise and enthusiasm to determining the effects of single-tracks of cosmic rays through biological systems. Your pioneering efforts in this emerging science of Space Radiation Biology will remain an inspiring example to us all.

\section{Acknowledgements}

This work is supported by the National Aeronautics and Space Administration under Order No. T-9310R, and by The NASA Specialized Center of Research and Training (NSCORT) awarded to the Lawrence Berkeley Laboratory/Colorado State University Consortium.

\section{References}

H. Bucker, The Biostack experiments I and II aboard Apollo 16 and 17, Life Sci. and Space Res. XII, 43-50 (1974).

S. B. Curtis and J. R. Letaw, Galactic cosmic rays and cell-hit frequencies outside the magnetosphere, Adv. Space Res. 9, (10)293-(10)298 (1989).

S. B. Curtis, L. W. Townsend, J. W. Wilson, P. Powers-Risius, E. L. Alpen, and R. J. M. Fry. Fluencerelated risk coefficients using the Harderian gland data as an example. Adv. Space Res. 12(No.23), (2)407-(2)416 (1992).

G. Horneck, Radiobiological experiments in space: A review, Nucl. Tracks Radiat. Meas. 20, 185-205 (1992).

J. R. Letaw, R. Silberberg and C. H. Tsao, Radiation hazards on space missions, Nature, 330, 709-710 (1987).

J. W. Wilson, J. L. Shinn and L W. Townsend, Nuclear reaction effects in conventional risk assessment for energetic ion exposure, Health Physics 58, $749-752$ (1990). 
Relative Effects of Hit to Non-hit Biological Objects in Space
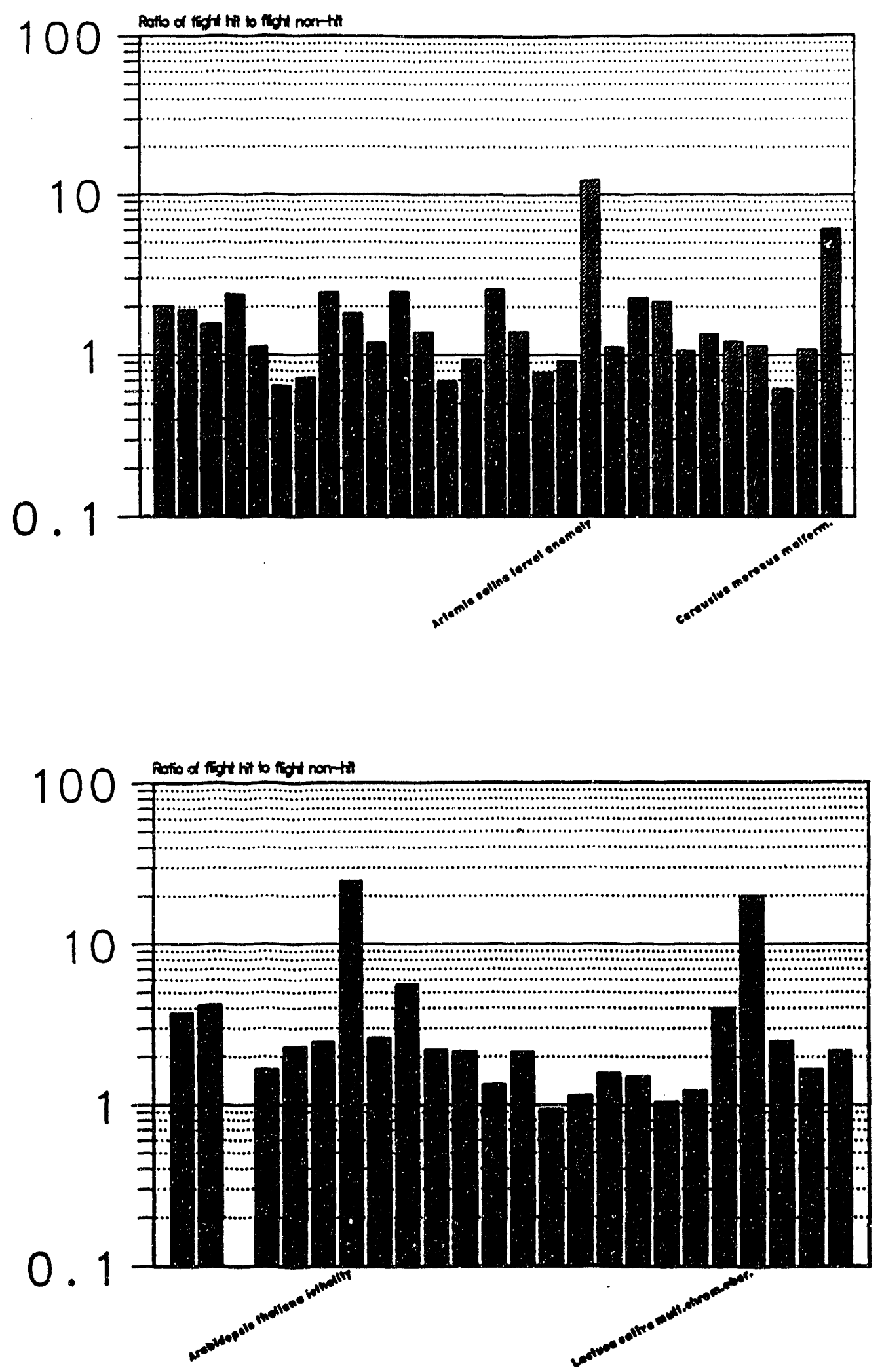

Figure 1. Ratios of biological effects from "hit" to "non-hit" samples from the galactic cosmic rays assuming a Poisson distribution as described in the text. A value of one means there was no difference in biological action measured between "hit" and "nonhit" samples. (From the data reviewed by Horneck, 19992). 

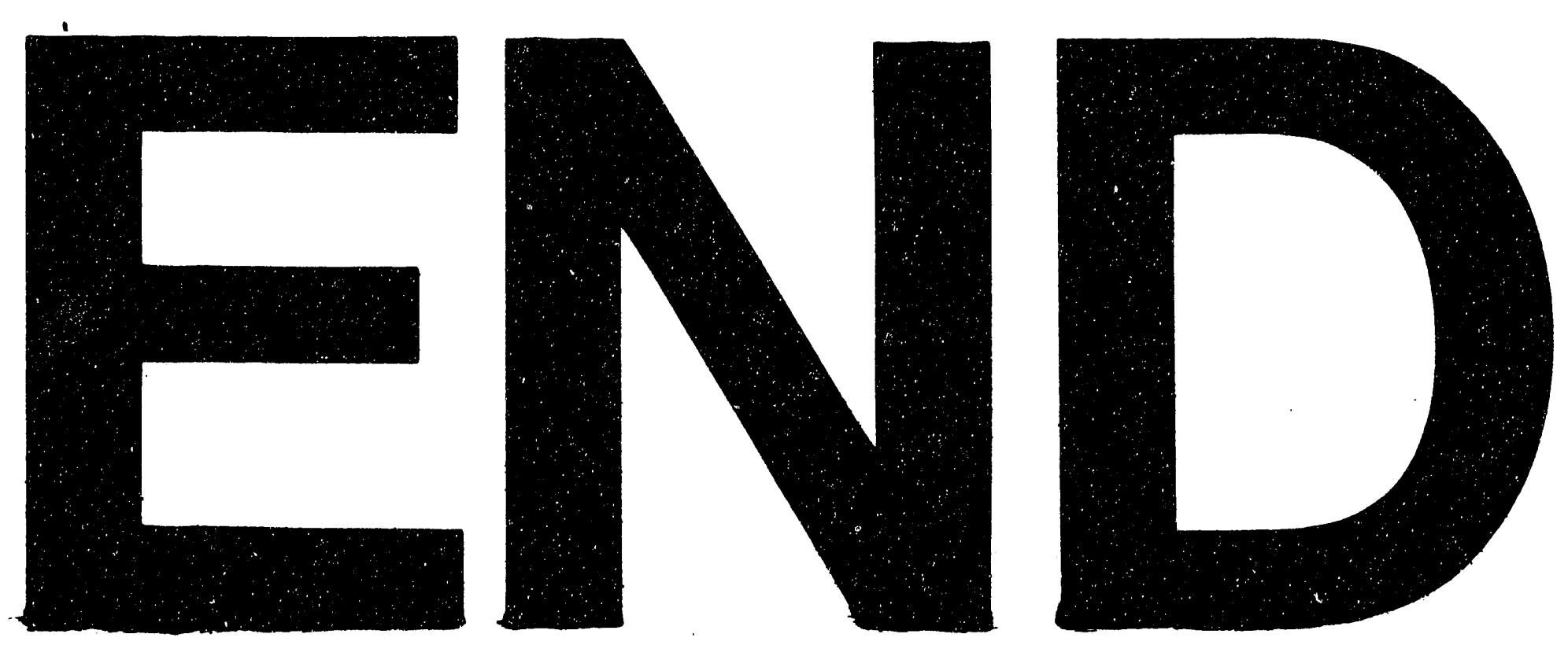

$x$
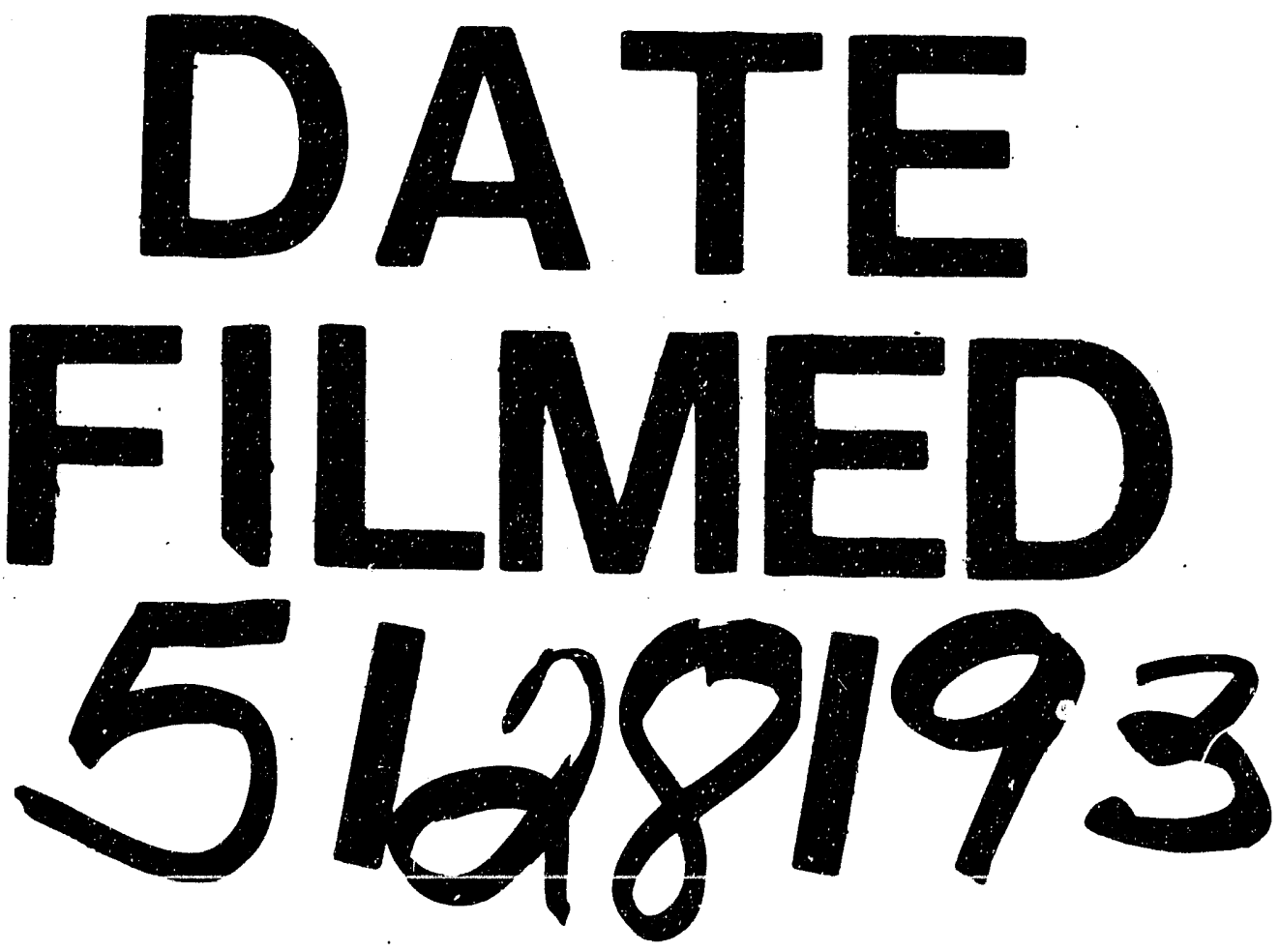
.

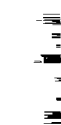

